\title{
Efeito da irrigação por aspersão no rendimento e potencial fisiológico das sementes de soja
}

\author{
Effect of sprinkler irrigation on yield and physiological potential of soybean seeds \\ Marciabela Fernandes Corrêa ${ }^{1}$, Renan Navroski ${ }^{1 *}$, Luis Osmar Braga Schuch ${ }^{1}$, Francisco de Jesus \\ Vernetti Junior ${ }^{2}$, Tainan Lopes de Almeida ${ }^{1}$, Hélen Claudine Saliba Rodrigues ${ }^{1}$, Ariele Paula Nadal ${ }^{1}$ e \\ Gizele Ingrid Gadotti ${ }^{1}$
}

${ }^{1}$ Universidade Federal de Pelotas, Pelotas, RS, Brasil. *Autor para correspondência: navroski@outlook.com.
${ }^{2}$ Embrapa Clima Temperado, Pelotas, RS, Brasil.

Submissão:27/06/2018 / Aceite: 01/07/2019

\begin{abstract}
RESUMO
A irrigação por aspersão pode minimizar os efeitos negativos da deficiência hídrica e aumentar o rendimento e desempenho de sementes de soja no campo. Assim, o objetivo do trabalho foi avaliar o efeito da irrigação por aspersão no rendimento e potencial fisiológico das sementes de soja produzidas em solos de várzea. Os tratamentos foram constituídos por oito cultivares de soja e dois regimes hídricos: com e sem irrigação por aspersão. Foram utilizadas cultivares de soja BMX Força RR, BRS Tertúlia RR, CD 249 RR STS, BMX Potência RR, do grupo de maturação (GM) 6 e BRS 246 RR, BRS Charrua RR, BRS Taura RR, e CD 231 RR, do grupo de maturação (GM) 7. As variáveis analisadas foram o rendimento de sementes, germinação, primeira contagem da germinação, envelhecimento acelerado, condutividade elétrica, emergência em campo e peso de mil sementes. A irrigação por aspersão na cultura da soja em solo de várzea proporciona acréscimos no rendimento de todas as cultivares em estudo, variando os acréscimos entre $32 \%$ a $139 \%$ e proporciona acréscimo no potencial fisiológico de sementes das cultivares BMX Força RR, BRS Tertúlia RR, CD 249 RR STS, BMX Potência RR, e BRS Taura RR.
\end{abstract}

PALAVRAS-CHAVE: Glycine max, déficit hídrico, germinação, solo de várzea.

\begin{abstract}
The sprinkler irrigation reduces the negative effects of water deficit and increases the yield and performance of soybean seeds on field. Thus, the objective of work was to evaluate the effect of sprinkler irrigation on yield and physiological potential of soybean seeds produced in lowland soils. The treatments consisted of 8 soybean cultivars and two water regimes: with and without sprinkler irrigation. This work was developed using soybean seeds of the cultivars BMX Força RR, BRS Tertúlia RR, CD 249 RR STS, BMX Potência RR and BRS 246 RR, BRS Charrua RR, BRS Taura RR, and CD 231 RR. The variables analyzed were the seeds yield, germination, first count of germination, accelerated aging, electrical conductivity, field emergence, and seed weight. The sprinkler irrigation on soybean crop in lowland soil increase in seed yield of all cultivars studied, the increase varied from $32 \%$ to $139 \%$. The sprinkler irrigation has improved seed performance of cultivars BMX Força RR, BRS Tertúlia RR, CD 249 RR STS, BMX Potência RR, and BRS Taura.
\end{abstract}

KEYWORDS: Glycine max, water deficit, germination, lowland soil.

\section{INTRODUÇÃO}

A cultura da soja é afetada pelo déficit hídrico e como forma de melhorar o desempenho de suas sementes e obter um estande adequado de plantas, a soja pode ser cultivada em área de várzea no Rio Grande do Sul, em rotação com a cultura do arroz sob o sistema de irrigação por aspersão.

Nos últimos anos o cultivo da soja tem apresentado maior expansão em ambientes onde predominam o cultivo de arroz irrigado e a pecuária de corte, estimando-se que $25 \%$ dessa área anualmente vem sendo destinada ao cultivo da soja em solos de várzea (MARCHESAN 2013).

No Rio Grande do Sul, o cultivo da soja ocupou uma área de 5,54 milhões de ha na safra 2016/2017. 
Apenas na região da Zona Sul do RS, região característica do cultivo de arroz em várzea, a área semeada com soja foi de 231.664 hectares na safra 2016/2017 (IBGE 2018). A produção de sementes de soja nestas regiões necessita ser explorada, visto que apenas $10 \%$ das sementes são produzidas nestas áreas (GAZOLLA NETO \& SCHUCH 2013).

A soja cultivada em área de várzea tem sido uma alternativa viável para maximizar o rendimento e o potencial fisiológico das sementes produzidas em função das frequentes estiagens ocorridas, no período do verão, no Sul do Brasil. A deficiência hídrica por estiagem neste período causa grandes prejuízos à agricultura, pois a chuva é a principal fonte de água para as culturas, e atinge diretamente 0 cultivo das culturas de grande importância econômica como a soja (COSTA et al. 2008). Na cultura da soja, o uso de irrigação pode minimizar os efeitos negativos da deficiência hídrica sobre o potencial fisiológico das sementes, segundo HEATHERLY (1993).

Para que as sementes de soja com desempenho superior atinjam seu potencial máximo em condições de campo, é necessária a utilização de tecnologias adequadas para maximizar a produção, estabelecimento do estande inicial e o desenvolvimento de plântulas. Esses fatores são essenciais para obter o rendimento de grãos esperado, mas para atender a esses requisitos é imprescindível a utilização de sementes com qualidade física e fisiológica superior. No entanto, mesmo com a utilização de sementes de alta qualidade, as condições climáticas adversas podem comprometer o estande inicial das plantas, principalmente quando a competição é maximizada por recursos como pela disponibilidade de água e nutrientes na lavoura (TAVARES et al. 2013).

A qualidade das sementes pode ser expressa como a soma dos atributos de pureza física, genética, sanitária e fisiológica. Dentre estes atributos, as qualidades física e fisiológica são de fundamental importância para estimar o potencial de desempenho das sementes no campo (TUNES et al. 2008). Os quatro componentes da qualidade de sementes possuem importância equivalente, mas o potencial fisiológico fornece informações sobre a germinação e vigor, pelo qual permite identificar lotes de sementes que possuem maior probabilidade de apresentar desempenho desejado durante 0 armazenamento e em campo (MARCOS FILHO 2013). Assim, o presente trabalho teve por objetivo avaliar o efeito da irrigação por aspersão sobre o rendimento e potencial fisiológico das sementes de soja produzidas em solos de várzea.

\section{MATERIAL E MÉTODOS}

O trabalho foi conduzido na Estação Experimental de Terras Baixas, da Embrapa Clima Temperado, localizada no Município de Capão do Leão, na safra agrícola 2011/2012, utilizando-se sementes de soja das cultivares BMX Força RR, BRS Tertúlia RR, CD 249 RR STS, BMX Potência RR do grupo de maturação 6 (ciclo médio), e sementes das cultivares BRS 246 RR, BRS Charrua RR, BRS Taura RR, e CD 231 RR do grupo de maturação 7 (ciclo tardio).

O experimento foi instalado na segunda quinzena do mês de novembro de 2011, em área de várzea, sob dois regimes hídricos: com e sem irrigação por aspersão, em um solo Planossolo Háplico Eutrófico solódico, com textura franco-arenosa de pouca profundidade e horizonte B impermeável.

A irrigação por aspersão foi realizada por meio do sistema linear móvel Valley, quando a tensão de umidade do solo atingiu $40 \mathrm{kPa}$. Foi aplicada uma lâmina total de irrigação de $147 \mathrm{~mm}$ durante o período de cultivo da soja. A precipitação pluvial total registrada durante o ciclo da cultura foi de $308 \mathrm{~mm}$, perfazendo dessa forma um total de $455 \mathrm{~mm}$.

O delineamento experimental utilizado foi de blocos ao acaso, com parcelas subdivididas compostas por quatro linhas de $5 \mathrm{~m}$ de comprimento, espaçadas em $0,5 \mathrm{~m}$ entre si. A área útil foi constituída das duas linhas centrais, eliminando-se $0,5 \mathrm{~m}$ de cada extremidade, perfazendo o total de $4 \mathrm{~m}^{2}$.

O rendimento de sementes foi obtido a partir da colheita manual da área útil das parcelas, sendo posteriormente trilhadas em trilhadeira estacionária. As sementes foram passadas em peneira com malha de $5 \mathrm{~mm}$, e após pesagem e correção para umidade de $13 \%$, os resultados foram expressos em kg ha-1. 0 peso de mil sementes (PMS) foi calculado a partir da contagem manual de oito repetições de 100 sementes cada. O resultado foi calculado multiplicando-se por 10 a massa média das oito repetições de 100 sementes, obtendo-se assim a massa de 1.000 sementes, conforme indicado pelas Regras de Análise de Sementes (MAPA 2009).

A amostra de trabalho foi obtida a partir das sementes colhidas em cada parcela do experimento, as sementes foram homogeneizadas e quarteadas para se obter a amostra de trabalho e o restante foi armazenado para eventual reteste.

Para as avaliações de qualidade fisiológica foram realizados os seguintes testes: Germinação $(G)$ : 
realizado com quatro repetições de 50 sementes, distribuídas em substrato de papel germitest, previamente umedecido com água destilada, utilizando a quantidade de água igual a 2,5 vezes a sua massa seca e colocados a uma temperatura constante de $25^{\circ} \mathrm{C}$. As avaliações e as contagens foram efetuadas ao quinto e oitavo dias após a semeadura (MAPA 2009). Primeira contagem da germinação (PCG): foram realizadas conjuntamente ao teste de germinação, aos cinco dias após início do teste; Envelhecimento acelerado (EA): as sementes foram distribuídas em camada única e uniforme sobre uma tela de alumínio suspensa no interior das caixas gerbox, contendo $40 \mathrm{~mL}$ de água deionizada e mantidas em câmara de germinação do tipo BOD a $41^{\circ} \mathrm{C}$, por 48 horas (MARCOS FILHO 1999). Após este período as sementes foram colocadas para germinar, conforme a metodologia descrita para o teste de germinação (MAPA 2009) e os resultados foram expressos em porcentagem de plântulas normais aos cinco dias de germinação; Condutividade elétrica (CE): foram utilizadas quatro repetições de 50 sementes, provenientes da porção de sementes puras. As sementes foram contadas e colocadas em copos plásticos de $200 \mathrm{~mL}$, contendo $75 \mathrm{~mL}$ de água deionizada, a temperatura de $20 \stackrel{\circ}{ } \mathrm{C}$ por 24 horas. Após este período realizou-se a leitura da condutividade elétrica da solução com condutivímetro digital. Os resultados foram expressos em microsiemens por centímetro $\left(\mu \mathrm{S} \mathrm{cm}^{-1}\right)$; Emergência em campo (EC): utilizaram-se quatro repetições de 50 sementes para cada unidade experimental. As avaliações foram realizadas aos 14 dias após a semeadura (TILLMANN \& MENEZES 2012). Os dados foram submetidos à análise da variância e as médias foram comparadas pelo teste de Scott-Knott, em nível de significância de 5\% de probabilidade.

\section{RESULTADOS E DISCUSSÃO}

Para o rendimento de sementes houve interação significativa entre o fator cultivar e o fator irrigação. $\mathrm{Na}$ Tabela 1, observa-se que todas as cultivares em estudo obtiveram aumento significativo no rendimento de sementes de soja devido irrigação por aspersão. As cultivares BMX Força RR e BRS Tertúlia RR do Grupo de Maturação (GM) 6, BRS 246 RR, BRS Charrua e CD 231 RR do GM 7 apresentaram rendimento superior a $2000 \mathrm{Kg} \mathrm{ha}^{-1}$, a BMX Potência RR e BRS Taura RR (GM7) apresentaram rendimento acima de $3000 \mathrm{Kg} \mathrm{ha}^{-1}$ quando irrigadas. Estes resultados assemelham-se aos obtidos por HERZOG et al. (2004), ao constatar rendimento médio de $3.334 \mathrm{~kg} \mathrm{ha}^{-1} \mathrm{em}$ condição de irrigação para as sementes de soja da cultivar Fepagro RS -10 comparado ao rendimento de $2.996 \mathrm{~kg} \mathrm{ha}^{-1}$ obtidos em ambiente sem irrigação.

Tabela 1. Rendimento em Kg ha-1 e peso de mil sementes em gramas de sementes de soja de diferentes cultivares submetidas a diferentes condições de irrigação.

Table 1. Yield in $\mathrm{kg} \mathrm{ha}^{-1}$ and weight of one thousand seeds in grams of soybean seeds of different cultivars under different irrigation conditions.

\begin{tabular}{|c|c|c|c|}
\hline \multicolumn{4}{|c|}{ Rendimento } \\
\hline Cultivar & Com irrigação & Sem irrigação & Incremento (\%) \\
\hline BMX Potência RR & $2.628 \mathrm{Aa}$ & $1.780 \mathrm{Ab}$ & 32 \\
\hline BMX Força RR & $2.432 \mathrm{Ba}$ & $1.542 \mathrm{Bb}$ & 37 \\
\hline BRS Tertúlia RR & $2.058 \mathrm{Ca}$ & $1.488 \mathrm{Bb}$ & 28 \\
\hline CD 249 RR STS & $1.700 \mathrm{Ca}$ & $725 \mathrm{Db}$ & 57 \\
\hline BRS 246 RR & $2.211 \mathrm{Ca}$ & $1.945 \mathrm{Aa}$ & 12 \\
\hline BRS Charrua RR & $1.955 \mathrm{Ca}$ & $1.190 \mathrm{Cb}$ & 39 \\
\hline BRS Taura RR & $2.831 \mathrm{Aa}$ & $1.281 \mathrm{Cb}$ & 55 \\
\hline CD 231 RR & $2.388 \mathrm{Ba}$ & $805 \mathrm{Db}$ & 66 \\
\hline Média & 2.275 & 1.345 & 40,75 \\
\hline \multicolumn{4}{|c|}{ Peso de mil sementes } \\
\hline BMX Potência RR & $16,75 \mathrm{Aa}$ & $13,78 \mathrm{Bb}$ & 18 \\
\hline BMX Força RR & $14,64 \mathrm{Ba}$ & $13,13 \mathrm{Cb}$ & 10 \\
\hline BRS Tertúlia RR & $15,26 \mathrm{Ba}$ & $12,90 \mathrm{Cb}$ & 15 \\
\hline CD 249 RR STS & $11,59 \mathrm{Da}$ & $11,19 \mathrm{Db}$ & 3 \\
\hline BRS 246 RR & $13,88 \mathrm{Ca}$ & $13,20 \mathrm{Cb}$ & 5 \\
\hline BRS Charrua RR & $12,86 \mathrm{Da}$ & $11,67 \mathrm{Db}$ & 9 \\
\hline BRS Taura RR & $16,30 \mathrm{Aa}$ & $15,43 \mathrm{Aa}$ & 5 \\
\hline CD 231 RR & $12,78 \mathrm{Da}$ & $12,61 \mathrm{Ca}$ & 1 \\
\hline Média & 14,26 & 12,99 & 9 \\
\hline
\end{tabular}

Mesmas letras maiúsculas não diferem entre cultivares e mesmas letras minúsculas não diferem entre os regimes hídricos significativamente pelo teste de Scott-Knott $(p \leq 0.05)$. 
De acordo com VERNETTI JUNIOR et al. (2011), a cultivar BRS 246 RR está entre as melhores opções de cultivares do Grupo de Maturação (GM) 7, para o sistema produtivo de Terras Baixas, pois em cinco anos de análise sempre produziu de $10 \%$ a $13 \%$ acima da média experimental. Em relação a BRS Tertúlia RR, VERNETTI JUNIOR \& NUNES (2010), observaram que a referida cultivar apresentou rendimento de grãos $6 \%$ superior à média geral do experimento de competição de cultivares do GM 6 . No entanto, neste trabalho destaca-se a cultivar CD 231 RR com acréscimo no rendimento de $139 \%$, seguida pelas cultivares BRS Taura RR com acréscimo de 136\% e CD 249 RR STS com 83\% de acréscimo no rendimento em função da irrigação por aspersão. Enquanto que a cultivar BRS 246 RR apresentou o menor acréscimo no rendimento com valor em torno de $32 \%$.

Em trabalhos realizados por KUSS et al. (2008), ao utilizar a irrigação por aspersão na cultura da soja em todo o ciclo, verificou-se um acréscimo no rendimento de sementes soja de $18 \%$ comparado ao tratamento sem irrigação. Nota-se na Tabela 1, que a cultivar BMX Potência RR mesmo quando submetida ao déficit hídrico obteve rendimento superior a $2000 \mathrm{Kg} \mathrm{ha}^{-1}$. Já as cultivares CD $231 \mathrm{RR}$ e CD 249 RR STS apresentaram os menores rendimentos em déficit hídrico, com rendimento de $1124 \mathrm{Kg} \mathrm{ha}^{-1} \mathrm{e} 1067 \mathrm{Kg} \mathrm{ha}^{-1}$, respectivamente. Estes baixos rendimentos se devem ao déficit hídrico ocorrido na safra 2011/2012, em que o rendimento de grãos teve uma redução de $45,3 \%$ em relação à safra anterior no estado do Rio Grande do Sul. A produtividade média no estado nesta safra foi de apenas $1555 \mathrm{Kg} \mathrm{ha}^{-1}$ (CONAB 2012), estando estes valores próximos aos encontrados neste trabalho na condição sem irrigação.

No peso de mil sementes houve um aumento significativo para as cultivares BMX Força RR, BRS Tertúlia RR, CD 249 RR, BMX Potência RR, BRS 246 RR e BRS Charrua RR no tratamento irrigado (Tabela 1). A irrigação por aspersão promoveu aumento no peso de mil sementes na média das cultivares em estudo, na ordem de $20 \mathrm{~g}$ em relação ao tratamento sem irrigação. De acordo com MAEHLER et al. (2003), as plantas de soja que receberam irrigação produziram grãos mais pesados, em média, o peso de 1000 grãos foi $33 \mathrm{~g}$ superior ao das plantas que não receberam irrigação.

As cultivares BMX Potência RR e BRS Taura RR que receberam irrigação apresentaram maior peso de mil sementes, bem como maior rendimento de sementes (Tabela 1). VERNETTI JUNIOR et al. (2011), encontrou resultados positivos no peso de mil sementes, resultando em acréscimos no rendimento de grãos de soja devido a irrigação.

Para THOMAS \& COSTA (2010), o rendimento é significativamente afetado quando ocorre o déficit hídrico durante o florescimento e no início da expansão dos legumes. De acordo com SALINAS et al. (1996), a ausência da irrigação no enchimento de grãos de soja reduz o tamanho e o peso de grãos em função da diminuição do suprimento de fotoassimilados produzidos pela planta e/ou inibição do metabolismo do próprio grão. A irrigação por aspersão na cultura da soja pode ser um benefício durante o enchimento de grãos porque a planta necessita de água nesse período para que não ocorra o abortamento das vagens e aumente o suprimento de fotoassimilados.

Trabalhos demonstram que a deficiência hídrica no desenvolvimento das sementes pode afetar além do rendimento, também a qualidade de sementes (CRUSCIOL et al. 2012). Para a qualidade de sementes houve aumento no percentual de germinação (Tabela 2) das sementes das cultivares BMX Força RR, BRS Tertúlia RR e BRS Taura RR no tratamento com irrigação. No entanto, SALINAS et al. (1996), ao produzir sementes de soja com e sem estresse hídrico não constataram aumento significativo no percentual de germinação das cultivares Forrest, Asgrow 5308, Federada I Inta, Pelotas 8549 e Pelotas 8546 sem estresse hídrico.

Pelos dados representados na Tabela 2, observa-se que a BRS Taura RR apresentou menor germinação que as demais cultivares em função da deficiência hídrica, destacando-se o aumento do percentual de germinação desta cultivar quando irrigada pelo sistema de aspersão em 11 pontos percentuais. Estes resultados estão de acordo com as afirmações realizadas por MOTA et al. (1996), ao relatar que a soja é uma cultura que necessita de irrigação em qualquer época de semeadura para todos os grupos de maturação, em todos os subperíodos e em todas as regiões climáticas do Rio Grande do Sul. Ressalta-se que as sementes de todas cultivares quando irrigadas por aspersão apresentaram germinação igual ou superior a $90 \%$.

No teste de primeira contagem da germinação (Tabela 2), as cultivares BMX Força RR, BRS Tertúlia RR, CD 249 RR STS, BRS 246 RR e BRS Taura RR apresentaram acréscimos no vigor devido à irrigação. As cultivares BMX Potência RR, CD 249 RR STS e BRS 246 RR apresentaram maior vigor que as demais cultivares no ambiente irrigado pelo sistema de aspersão. Observa-se na Tabela 2, que a BRS Tertúlia RR e BRS Taura RR obtiveram vigor de $78 \%$ e $71 \%$ em condição de déficit hídrico. Condições ambientais desfavoráveis durante o desenvolvimento e maturação de sementes de soja reduz a viabilidade e o vigor 
das mesmas (DORNBOS et al. 1989).

Tabela 2. Primeira contagem e germinação de sementes de soja de diferentes cultivares submetidas a diferentes condições de irrigação.

Table 2. First counting and germination of soybean seeds of different cultivars under different irrigation conditions.

\begin{tabular}{lcc}
\hline & $1 \stackrel{\text { a } \text { Contagem }}{ }$ \\
\hline Cultivar & Com irrigação & Sem irrigação \\
\hline BMX Potência RR & $90 \mathrm{Aa}$ & $88 \mathrm{Aa}$ \\
BMX Força RR & $83 \mathrm{Ba}$ & $80 \mathrm{Bb}$ \\
BRS Tertúlia RR & $85 \mathrm{Ba}$ & $78 \mathrm{Bb}$ \\
CD 249 RR STS & $94 \mathrm{Aa}$ & $86 \mathrm{Bb}$ \\
BRS 246 RR & $88 \mathrm{Aa}$ & $83 \mathrm{Bb}$ \\
BRS Charrua RR & $85 \mathrm{Ba}$ & $87 \mathrm{Aa}$ \\
BRS Taura RR & $85 \mathrm{Ba}$ & $74 \mathrm{Cb}$ \\
CD 231 RR & $83 \mathrm{Ca}$ & $80 \mathrm{Ba}$ \\
\hline & Germinação & \\
\hline BMX Potência RR & $96 \mathrm{Aa}$ & $92 \mathrm{Aa}$ \\
BMX Força RR & $91 \mathrm{Ba}$ & $85 \mathrm{Bb}$ \\
BRS Tertúlia RR & $93 \mathrm{Ba}$ & $83 \mathrm{Bb}$ \\
CD 249 RR STS & $97 \mathrm{Aa}$ & $94 \mathrm{Aa}$ \\
BRS 246 RR & $91 \mathrm{Ba}$ & $91 \mathrm{Aa}$ \\
BRS Charrua RR & $90 \mathrm{Ba}$ & $92 \mathrm{Aa}$ \\
BRS Taura RR & $90 \mathrm{Ba}$ & $81 \mathrm{Cb}$ \\
CD 231 RR & $89 \mathrm{Ba}$ & $88 \mathrm{Aa}$ \\
\hline
\end{tabular}

Mesmas letras maiúsculas não diferem entre cultivares e mesmas letras minúsculas não diferem entre os regimes hídricos significativamente pelo teste de Scott-Knott $(p \leq 0.05)$.

No teste de condutividade elétrica (Tabela 3), as cultivares BRS Tertúlia RR, CD 249 RR STS e BRS Taura RR apresentaram baixos valores de condutividade elétrica ou maior vigor em sementes oriundas da irrigação por aspersão. No entanto, ao comparar os resultados de condutividade elétrica (Tabela 3) das sementes no ambiente irrigado observou-se que todas as cultivares em estudo, não diferiram entre si. Mas, quando as mesmas cultivares foram submetidas ao déficit hídrico, verificou-se que a CD 249 RR STS apresentou maior lixiviação ou menor vigor comparado às demais, as quais não diferiram estatisticamente entre si para o teste de condutividade elétrica. Isso evidencia que o tratamento de irrigação por aspersão manteve a integridade das membranas celulares das sementes de soja da maioria das cultivares em estudo, o que possivelmente o déficit hídrico pode ter afetado mais acentuadamente a integridade das membranas celulares das sementes da cultivar CD 249 RR STS. Estes resultados assemelham-se aos obtidos por CRUSCIOL et al. (2012), ao verificar valores de condutividade elétrica significativamente superiores oriundos de sementes de arroz cultivado pelo sistema de sequeiro em relação as sementes provenientes do tratamento de irrigação por aspersão.

MARCOS FILHO (2005) relata que as sementes mais vigorosas, ou menos deterioradas são aquelas que apresentam maior velocidade de reestabelecimento da integridade das membranas celulares durante a embebição e consequentemente liberam menores quantidades de solutos para o meio exterior. Analisando os dados obtidos nesta avaliação, verifica-se que os menores valores de condutividade elétrica para as sementes produzidas sob o sistema de irrigação por aspersão foi de $72,9 \mu \mathrm{S} . \mathrm{cm}^{-1}$ para BRS Tertúlia RR e o maior foi em torno de $90,4 \mu \mathrm{S} . \mathrm{cm}^{-1}$ para a cultivar CD $231 \mathrm{RR}$, sendo que para as sementes oriundas do tratamento por déficit hídrico, o menor valor foi de $81.6 \mu \mathrm{S} . \mathrm{cm}^{-1}$ para BMX potência RR e o maior de 140.7 $\mu S . \mathrm{cm}^{-1}$ para CD 249 RR STS. De acordo com AOSA (2002), sementes de soja com condutividade elétrica até $60-70 \mu \mathrm{S} . \mathrm{cm}^{-1} \cdot \mathrm{g}^{-1}$, são consideradas de alto vigor, enquanto $70-80 \mu \mathrm{S} . \mathrm{cm}^{-1} \cdot \mathrm{g}^{-1}$ são valores com tendência para médio vigor. Já, VIEIRA et al. (2004), consideram que sementes com valores de até $90 \mu \mathrm{S} . \mathrm{cm}^{-1} \cdot \mathrm{g}^{-1}$ podem apresentar desempenho satisfatório em condições de campo abaixo do ideal.

Para a emergência em campo, podemos destacar que a irrigação por aspersão causou efeito positivo no potencial fisiológico da semente das cultivares BMX Força RR, BRS Tertúlia RR, CD 249 RR STS, BMX Potência RR e BRS Taura RR (Tabela 3). Segundo CRUSCIOL et al. (2002), o sistema irrigado por aspersão favoreceu a produção de sementes de arroz de maior vigor.

No teste de envelhecimento acelerado (Tabela 4), observa-se que não houve interação entre os fatores cultivar e tratamento com e sem irrigação por aspersão. Embora, não tenha ocorrido interação entre 
os fatores analisados, o vigor médio das sementes oriundas de todas as cultivares submetidas à irrigação foi superior à média das cultivares que não receberam irrigação no teste de envelhecimento acelerado. Estes resultados estão de acordo aos encontrados por CRUSCIOL et al. (2002), em que detectou maior percentual de plântulas normais no teste de envelhecimento acelerado quando foi usado o sistema de irrigação por aspersão. Nos resultados obtidos por SILVA et al. (2007), as sementes de soja das cultivares Conquista e IAC 8-2 produzidas em área sem suplementação hídrica obtiveram valores de 84 e 95\% no teste de envelhecimento acelerado. DORNBOS et al. (1989), constatou que em sementes de soja são relatadas ocorrências de redução da germinação e do vigor como consequência da deficiência hídrica. A disponibilidade hídrica é um fator limitante ao rendimento da cultura, visto que a precipitação não atende à demanda potencial de água para o cultivo de soja (MOTA et al. 1996).

Tabela 3. Condutividade elétrica e emergência em campo de sementes de soja de diferentes cultivares submetidas a diferentes condições de irrigação.

Table 3. Electrical conductivity and field emergence of soybean seeds of different cultivars under different irrigation conditions.

\begin{tabular}{lcc}
\hline & Condutividade elétrica & \\
\hline Cultivar & Com irrigação & Sem irrigação \\
\hline BMX Potência RR & $75,82 \mathrm{Ab}$ & $79,65 \mathrm{Aa}$ \\
BMX Força RR & $77,73 \mathrm{Aa}$ & $90,92 \mathrm{Ba}$ \\
BRS Tertúlia RR & $72,95 \mathrm{Ab}$ & $9780 \mathrm{Ba}$ \\
CD 249 RR STS & $74,70 \mathrm{Ab}$ & $140,72 \mathrm{aA}$ \\
BRS 246 RR & $83,91 \mathrm{Aa}$ & $85,75 \mathrm{Ba}$ \\
BRS Charrua RR & $85,93 \mathrm{Aa}$ & $87,10 \mathrm{Ba}$ \\
RS Taura RR & $81,67 \mathrm{Ab}$ & $106,85 \mathrm{Ba}$ \\
CD 231 RR & $90,48 \mathrm{Aa}$ & $100,17 \mathrm{Ba}$ \\
\hline & Emergência & \\
\hline BMX Potência RR & $89 \mathrm{Aa}$ & $84 \mathrm{Ba}$ \\
BMX Força RR & $91 \mathrm{Aa}$ & $86 \mathrm{Ba}$ \\
BRS Tertúlia RR & $93 \mathrm{Aa}$ & $83 \mathrm{Bb}$ \\
CD 249 RR STS & $89 \mathrm{Aa}$ & $90 \mathrm{Aa}$ \\
BRS 246 RR & $89 \mathrm{Aa}$ & $90 \mathrm{Aa}$ \\
BRS Charrua RR & $96 \mathrm{Aa}$ & $95 \mathrm{Aa}$ \\
RS Taura RR & $82 \mathrm{Ba}$ & $86 \mathrm{Ba}$ \\
CD 231 RR & $81 \mathrm{Ba}$ & $88 \mathrm{Aa}$ \\
\hline
\end{tabular}

Mesmas letras maiúsculas não diferem entre cultivares e mesmas letras minúsculas não diferem entre os regimes hídricos significativamente pelo teste de Scott-Knott $(p \leq 0.05)$.

Tabela 4. Envelhecimento acelerado em sementes de soja de diferentes cultivares submetidas a diferentes condições de irrigação.

Table 4. Accelerated aging in soybean seeds of different cultivars under different irrigation conditions.

\begin{tabular}{lcc}
\hline Cultivar & Com irrigação & Sem irrigação \\
\hline BMX Potência RR & A & B \\
BMX Força RR & $80^{\text {ns }}$ & 75 \\
BRS Tertúlia RR & 81 & 60 \\
CD 249 RR STS & 75 & 60 \\
BRS 246 RR & 78 & 71 \\
BRS Charrua RR & 86 & 83 \\
RS Taura RR & 82 & 48 \\
CD 231 RR & 83 & 54 \\
\hline
\end{tabular}

Mesmas letras maiúsculas não diferem entre os regimes hídricos significativamente pelo teste de Scott-Knott ( $p \leq 0.05)$. ns $=$ não significativo nas colunas, entre cultivares.

No presente trabalho, a irrigação por aspersão realizada na cultura da soja aumentou o rendimento e a qualidade das sementes de soja produzida em solos de várzea. Com o aumento da área semeada da soja em solos de várzea será necessário aumentar a produção de sementes de alta qualidade, além de ser uma alternativa ao cultivo sucessivo de arroz irrigado. 


\section{CONCLUSÃO}

A irrigação por aspersão na cultura da soja em solo de várzea proporciona acréscimos no rendimento de sementes das cultivares em estudo de ambos os grupos de maturação e melhora o desempenho de sementes das cultivares BMX Força RR, BRS Tertúlia RR, CD 249 RR STS, BMX Potência RR, e BRS Taura RR.

\section{REFERÊNCIAS}

AOSA. 2002. Association of Official Seed Analysts. Seed vigor testing handbook. Lincoln: AOSA. 105p. (Contribuition, 32).

CONAB. 2012. Companhia Nacional de Abastecimento. Acompanhamento da safra brasileira de grãos: nono levantamento. Brasília: CONAB. 34p.

COSTA RCL. et al. 2008. Biochemical and physiological responsesin two Vigna unguiculata (L.) Walp. cultivars under waterstress. Journal of Agronomy 7: 98-101.

CRUSCIOL CAC et al. 2002. Produtividade e qualidade fisiológica de sementes de duas cultivares de arroz de terras altas em dois sistemas de cultivo. Revista Acta Scientiarum 24: 1569-1574.

CRUSCIOL CAC. et al. 2012. Fornecimento de água por meio de irrigação por aspersão para produção de sementes de arroz de terras altas. Bioscience Journal 28: 34-42.

DORNBOS DL et al. 1989. Drought stress effects during seed fill on soybean seed germination and vigor. Crop Science 29: 476-480.

GAZOLLA NETO A \& SCHUCH LOB. 2013. Produção de semente e o avanço da soja na Metade Sul do Rio Grande do Sul. Seed News 17: 36-37.

HEATHERLY LG. 1993. Drought stress and irrigation effects on germination of harvested soybean seed. Crop Sciense 33: 777-781.

HERZOG RLS et al. 2004. Produtividade de soja em semeadura direta influenciada por profundidade do sulcador e adubo e doses de resíduos em sistema irrigado e não irrigado. Engenharia Agrícola 24: 771-780.

INSTITUTO BRASILEIRO DE GEOGRAFIA E ESTATÍSTICA - IBGE. 2018. Produção agrícola municipal. Rio de Janeiro: IBGE. Available at: http://www.sidra.ibge.gov.br/tabela/1612. Accessed at: 09 mar. 2018.

KUSS RCR et al. 2008. Populações de plantas e estratégias de manejo de irrigação na cultura da soja. Ciência Rural 38: 1133-1137

MAEHLER AR et al. 2003. Potencial de rendimento da soja durante a ontogenia em razão da irrigação e arranjo de plantas. Pesquisa Agropecuária Brasileira 38: 225-231.

MAPA. 2009. Ministério da Agricultura, Pecuária e Abastecimento. Regras para análise de sementes. Brasília: MAPA/ACS. 399p.

MARCHESAN E. 2013. Desafios e perspectivas de rotação com soja em áreas de arroz. Disponível em: http://cbai2013.web2265.uni5.net/docs/cbai2013_palestra_enio_marchesan.pdf. Acesso em: 4 mai 2015.

MARCOS FILHO J. 2005. Fisiologia de sementes de plantas cultivadas. Piracicaba: FEALQ. 495p.

MARCOS FILHO J. 1999. Teste de envelhecimento acelerado. In: KRZYZANOWSKI FC et al. (Ed.). Vigor de sementes: conceitos e testes. Londrina: ABRATES. p.1-24.

MARCOS FILHO J. 2013. Importância do potencial fisiológico de sementes de soja. Informativo Abrates 23: 21-24.

MOTA FS et al. 1996. Análise agroclimática da necessidade de irrigação da soja no Rio Grande do Sul. Revista Brasileira de Agrometeorologia 4: 133-138.

SALINAS AR et al. 1996. Respuestas fisiológicas que caracterizan el comportamiento de diferentes cultivares de soja a la deficiencia hídrica en el suelo. Pesquisa Agropecuaria Brasileira 31: 331-338.

SILVA JB et al. 2007. Avaliação de genótipos de soja em semeadura de inverno, em Selvíria, MS: produção e qualidade fisiológica de sementes. Revista Brasileira de Sementes 29: 169-176.

TAVARES LC et al. 2013. Desempenho de sementes de soja sob deficiência hídrica: rendimento e qualidade fisiológica da geração F1. Ciência Rural 43: 1357-1363.

THOMAS AL \& COSTA JA. 2010. Estresse hídrico em soja: impacto no potencial de rendimento de grãos. In: THOMAS AL \& COSTA JA. Soja: manejo para alta produtividade de grãos. Porto Alegre: Evangraf. 248p.

TILLMANN MÂA \& MENEZES NL. 2012. Análise de sementes. In: PESKE ST et al. Sementes: fundamentos científicos e tecnológicos. 3.ed. Pelotas: UFPel. p.161-272.

TUNES LM et al. 2008. Testes de vigor em sementes de aveia branca. Revista da FZVA 15: 94-106.

VERNETTI JUNIOR FJ \& NUNES TL. 2010. Avaliação de cultivares da rede soja sul de pesquisa do grupo de maturidade seis tolerantes ao glifosato, na Embrapa Clima Temperado. Pelotas: Embrapa. p. 17-22. (Documentos 322).

VERNETTI JUNIOR FJ. et al. 2011. Cultivares de soja num sistema de produção com arroz, irrigados por aspersão. Resultados de pesquisa de soja na Embrapa Clima Temperado. Pelotas: Embrapa. p.61-66. (Documentos, 340).

VIEIRA RD et al. 2004. Electrical conductivity of the seed soaking solution and soybean seedling emergence. Scientia Agricola 61: 164-168. 